\title{
Heme Binding in Gas-Phase Holo-Myoglobin Cations: Distal Becomes Proximal?
}

\author{
Atim A. Enyenihi, ${ }^{1}$ Hongqian Yang, ${ }^{1}$ A. Jimmy Ytterberg, ${ }^{1}$ Yaroslav Lyutvinskiy, ${ }^{1}$ \\ Roman A. Zubarev ${ }^{1,2}$ \\ ${ }^{1}$ Division of Physiological Chemistry I, Department of Medical Biochemistry and Biophysics, Karolinska Institutet, \\ Scheelesväg 2, SE-17 177 Stockholm, Sweden \\ ${ }^{2}$ Science for Life Laboratory, Stockholm, Sweden
}

\begin{abstract}
His64 and His93 are the two well-known sites of heme binding in water-dissolved holomyoglobin, with His93 being a proximal, strongly binding partner, while the distal His64 weakly coordinates to the heme through a small-molecule ligand, e.g., water or $\mathrm{O}_{2}$. The heme bonding scheme in a water-free environment is as yet unclear. Here we employed electron transfer dissociation tandem mass spectrometry to study the preferential attachment site of the ferriheme $\left(\mathrm{Fe}^{3+}\right)$ in electrospray-produced $12+, 14+$, and $16+$ holo-myoglobin ions. Contrary to expectations, in lower-charge complexes that should have a structure resembling that in solution, the heme seems to be preferentially attached to the "distal" histidine. In contrast, in the highest studied charge state, the "proximal" histidine is the site of preferential attachment; the 14 + charge state is an intermediate case. This surprising finding raises a question of heme coordination in proteins transferred to water-free environment, as well as the effect of the protonation sites on heme bonding.
\end{abstract}

Key words: Electron transfer dissociation, Myoglobin, Supercharging, Top-down mass spectrometry, Proteomics, Non-covalent interactions, Proteins, Top-down protein complex analysis

\section{Introduction}

$\mathrm{M}$ yoglobin $(\mathrm{Mb})$ is a small heme-containing monomeric enzyme found in the muscle cells of all vertebrates. It is the first protein whose three-dimensional structure was successfully determined (1958) by high-resolution X-ray crystallography [1]. Despite being one of the most studied proteins in biology, myoglobin still presents surprises, and the structures of its wild type and numerous mutants are still under detailed investigation.

Electronic supplementary material The online version of this article (doi:10.1007/s13361-011-0182-0) contains supplementary material, which is available to authorized users.

Correspondence to: Roman A. Zubarev; e-mail: Roman.Zubarev@ki.se
In solution, the myoglobin polypeptide chain (apomyoglobin, $\mathrm{aMb}$ ) has an extremely high affinity for heme $\left(\sim 3 \cdot 10^{14} \mathrm{M}^{-1}\right)$, and readily forms an aMb-heme complex (holo-myolgobin, hMb). Myoglobin heme is a prosthetic group (cofactor tightly bound to the enzyme) made up of protoporphyrin IX that coordinates an iron atom. The bonding to the $\mathrm{aMb}$ polypeptide chain occurs via two histidine resides, His64 and His93 (Figure 1). The bonding and role of these two sites are not equivalent. His 93 is a proximal histidine group axially coordinated to the iron center (Figure 1a). The heme group is not completely flat: the iron atom is about $0.3 \mathrm{~A}$ out of the plane of the porphyrin, on the same side as the proximal histidine. The bond between the heme and proximal histidine is so strong that it is sometimes called covalent [2]. On the opposite face of the heme, there is a distal histidine, His64, which is not bonded directly to the iron. Instead, the second axial ligand 
(a)

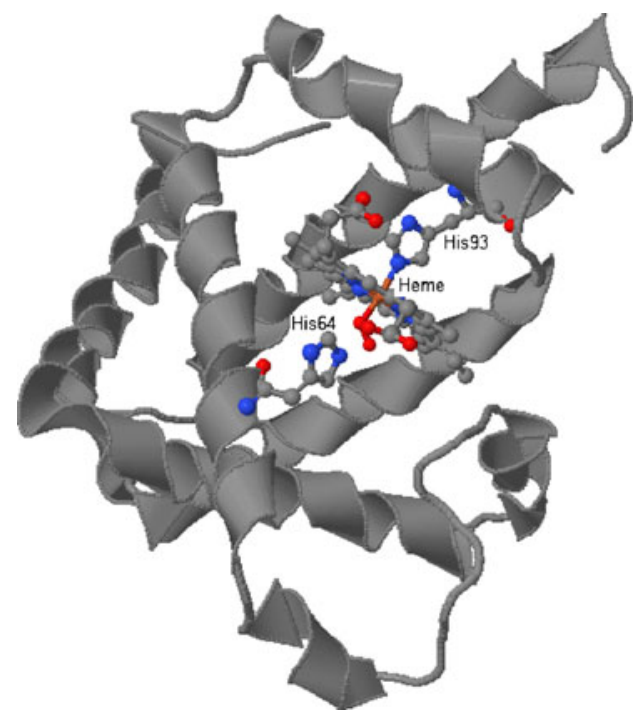

(b)

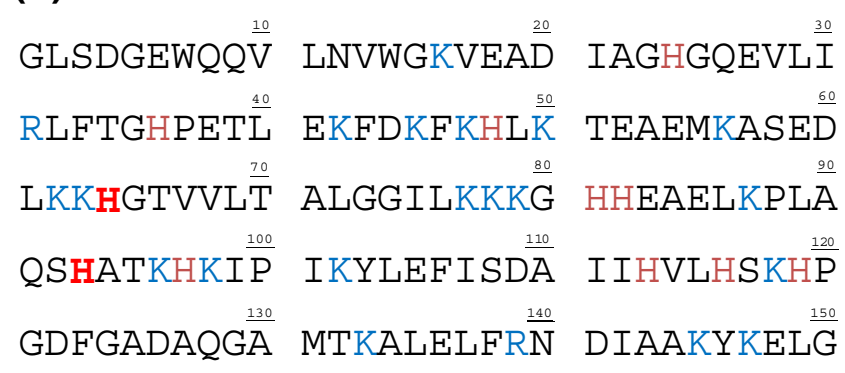

FQG

Figure 1. (a) Structure of holo-myoglobin showing the heme position between histidines 64 and 93. (b) Sequence of myoglobin; distal and proximal histidines are shown in red. The basic amino acid residues (potential protonation sites) are shown in blue; histidine residues (potential sites for heme attachment) are in brown

of the hemin iron atom is either exogenous water in the ferric state or $\mathrm{O}_{2}$ when the iron is reduced. Both of these ligands are indirectly attached to the protein by hydrogen bonding to the distal histidine [3]. The ligand is relatively strongly bound, but it can be removed under certain circumstances. In the absence of ligand in the heme pocket, the distal pocket will remain vacant. It has been shown that in some circumstances, iron will coordinate to both the proximal and distal histidine residues [4] Depending on the oxidation state of the iron, these bishistidine complexes are called hemichrome [Fe(III)] or hemochrome [Fe(II)]. Hemichrome states of native $\mathrm{hMb}$ have been considered as precursors of denaturation processes, such as unfolding, precipitation, and heme dissociation (the presence of hemichromes in blood is associated with heart disease), but recently, hemichromes were found in native-like hemoglobin structures [5].

For a long time, the function of $\mathrm{Mb}$ was believed to be intimately linked to the water environment. However, recently it has been shown that gradual removal of water from myoglobin solution and replacing it with polymer surfactants largely preserves the native fold of $\mathrm{hMb}$ and its ligand-binding function [6]. This result gave a reason to rethink the role of water in myoglobin activity, and reinstated interest in studying details of the $\mathrm{hMb}$ structure in water-free environment.

To be fair, the surfactant replacing technique left approximately four $\mathrm{H}_{2} \mathrm{O}$ molecules bound on the surface of each $\mathrm{Mb}$ molecule, including the water molecule in the distal heme pocket [6]. In contrast to water-surfactant replacement, electrospray ionization (ESI) produces completely water-free gas-phase $\mathrm{hMb}$ ions even without extensive heating. The properties of gas-phase $\mathrm{hMb}$ ions have been studied by mass spectrometry since $1988[7,8]$. Of particular interest was to determine whether or not the solution-phase structure is retained in the gas phase. The starkest structural feature of $\mathrm{hMb}$ is the heme noncovalently bonded to the $\mathrm{aMb}$ chain. To establish that the heme is still attached to the $\mathrm{aMb}$ chain is trivial in mass spectrometry; the real question is whether or not the attachment site(s) is/are the same as in solution.

In one work [9], Hunter et al. studied a series of mutant myoglobins where individual solution-phase hydrogen bonds between the heme and protein were systematically removed. Collisional dissociation of $11+$ to $14+\mathrm{hMb}$ ions in the orifice-skimmer region of an ESI mass spectrometer showed a strong correlation between the threshold dissociation voltages and the heme binding energies in solution. It was concluded that the heme binding pocket in gas phase hMB ions retains much of its solution structure. Continuing that line of work, Chen et al. studied dissociation of $\mathrm{hMb}$ complexes in charge states $7+$ to $21+[10]$. Collision cross section measurements showed partial unfolding of the highly charged ions compared with the low charge states, but the energies required to dissociate heme from the highly charged heme-protein complexes were found similar to those of low charge states. The results were interpreted in support of preservation of the solution-phase heme-protein interactions even in the highly charged gas phase hMb ions.

In contrast, Schmidt and Karas have found the extent of dissociation of the $\mathrm{hMb}$ non-covalent complexes upon collisional activation and, thus, their gas-phase stabilities, is strongly dependent on the polarity of the ESI-MS experiment as well as on the charge of the heme prosthetic group [11]. They concluded that Coulombic interaction between the charged heme and the positive or negative charges on the $\mathrm{aMb}$ chain are important for the $\mathrm{hMb}$ complex stability. Mark and Douglas have largely agreed with this conclusion, having found that the collisional energies required to induce charged heme loss from $4+$ to $8+$ positive $\mathrm{hMb}$ ions are significantly lower than from 4- to 7- $\mathrm{hMb}$ negative ions [12]. They explained the results by a simple model that lowers the barrier for dissociation due to the Coulombic interaction between the charged heme attached to the proximal histidine and other charged sites in the $\mathrm{hMb}$ complex. Recently, Lomeli et al. $[13,14]$ have shown that super-charging of $\mathrm{hMb}$ and other non-covalent complexes in positive ESI by adding certain small molecules 
to the ESI solution does not lead to dissociation of the complexes. Sterling et al. [15] have studied the supercharged $\mathrm{hMb}$ structure and found partial unfolding that, however, did not result in a heme loss.

The critical question that has not been addressed so far in the literature is whether or not the heme in gas-phase $\mathrm{hMb}$ ions remains five-coordinated as in the native structure or six-coordinated as in bishistidine hemichromes. From solution studies it follows that in the absence of the distal water or other ligand, the distal histidine in native $\mathrm{hMb}$ can be coordinated to the heme, creating a hemichrome structure. Therefore, such a structure may be present or even dominate in the gas phase. But if the heme is bonded to two histidines, the question is whether the bond to proximal histidine is stronger, as in solution.

Here we employed tandem mass spectrometry (MS/MS) to address these questions. Unlike collisional and infrared dissociation employed in the previous studies, fragmentation reactions involving capturing an electron by polypeptide polycations, such as electron capture dissociation (ECD [16]) and electron transfer dissociation (ETD [17]), preserve at certain conditions non-covalent interactions. This has been demonstrated a decade ago on a peptide-peptide complex [18]. Loo et al. have extended this approach to a complex involving a full-size protein [19]. In the previous work on a 7 tesla LTQ FT (ThermoFisher, Bremen, Germany) mass spectrometer, we have shown that electron ionization dissociation (EID), an ion-electron reaction with $>40 \mathrm{eV}$ electrons, can locate the heme in $\mathrm{hMb}$ with the accuracy of four-five residues [20]. Using another platform (ETDcapable Orbitrap Velos, ThermoFisher, Bremen, Germany), we have obtained high-quality ETD MS/MS spectra of hMb and $\mathrm{aMb}$ in different charge states and investigated the question of the heme binding position in more detail.

It is understood that the heme binding position may depend upon the protonation state of the gas-phase molecule, as abundant protonation destabilizes the "native" structure even in non-covalent complexes [15]. This is why most studies on "native"-like gas-phase protein structures are performed on relatively low charge states [20]. However, low charge states mean a high $\mathrm{m} / \mathrm{z}$, at which FT mass analyzers lose their high resolving power. The efficiency of electron-capture based fragmentation techniques also usually suffers as the charge state of the precursors decreases. Thus, the challenge is to achieve as high a protonation level of $\mathrm{hMb}$ as possible without losing the similarity between the gas-phase structure and the solution structure. Recently, the Loo group has reported that the addition of certain agents to the electrospray solvent achieves super-charging of proteinmolecule complexes without their dissociation [13, 14]. Thus, we performed ETD MS/MS on the most abundant charge state achievable with the "native solution" $(12+)$, and its super-charged counterpart, 16+ (Figure 2), as well as the intermediate charge state $14+$. We found that the solutionphase heme location between the histidines 64 and 93 is largely preserved in the gas phase even at super-charging.
However, the heme preferential bonding is not necessarily to the proximal histidine. Therefore, the relevance of gas-phase data to solution phase is not straightforward and requires careful consideration.

\section{Experimental}

Myoglobin from horse skeletal muscle was purchased from Sigma-Aldrich, Inc. and used without further purification. The protein was dissolved in an aqueous solution of $3 \%$ methanol/ $/ 0.5 \mathrm{mM}$ ammonium acetate, $\mathrm{pH} \sim 6.8$ and to a concentration of $\sim 5 \mu \mathrm{M}$ and electrosprayed from a metalcoated pulled-glass capillary (Proxeon, Odense, Denmark). For super-charging, $5 \mathrm{mM}$ sulfolane was added to an aqueous solution of myoglobin in $30 \%$ acetonitrile. The final concentration of Mb was $5 \mu \mathrm{M}$. The mass spectrometry experiments were performed on an LTQ Orbitrap Velos instrument with ETD (ThermoFisher Scientific). The ETD conditions were optimized for each charge state. Reaction times ranged from 4 to $15 \mathrm{~ms}$. Fragment assignment was performed using in-house software written in Perl. Each detected fragment was inspected; its charge state and massto-charge ratio were validated manually.

\section{Results}

Figure 2a shows the electrospray mass spectrum of myoglobin. Under the spraying conditions, some loss of the heme occurs, as evident from the $\mathrm{aMb} \mathrm{a}^{10+}-\mathrm{a}^{21+}$ ions. However, the most abundant ions are those of intact $\mathrm{hMb}$, labeled $\mathrm{h}^{9+}$ $\mathrm{h}^{14+}$. No evidence of water retention in $\mathrm{hMb}$ ions is observed. Addition of $5 \mathrm{mM}$ sulfolane to $\mathrm{hMb}$ solution (Figure $2 b$ ) shifts the highest protein charge state from 14+ to $19+$ while $\mathrm{hMb}$ retains the heme.

Charge states $12+, 14+$, and $16+$ of $\mathrm{hMb}$ were isolated for ETD MS/MS in the linear ion trap. Neutral mass deconvolution confirmed that the heme is in a ferric state $\left(\mathrm{Fe}^{3+}\right)$ for these three charge states (Supplementary Materials). Moreover, deconvolution of the charge-reduced species revealed the same neutral mass as for the precursor species for both $\mathrm{hMb}$ and $\mathrm{aMb}$. Therefore, in non-dissociative electron capture, one proton gets reduced by electron and leaves as a neutral hydrogen atom, while heme in the charge-reduced species retains its charge state.

The ETD MS/MS spectrum of the 12+ charge state of $\mathrm{hMb}$ is shown in Figure 3a. The isotopic distributions of the shortest heme-containing fragments of both c- and z-type ions are shown in insets. Figure $3 \mathrm{~b}$ summarizes the backbone cleavages found in the spectrum. These fragments locate the heme attachment site to between the residues 63 and 77 according to the observed ions $\left[\mathrm{z}_{90}+\text { heme }\right]^{5+}$ and $\left[\mathrm{c}_{77}+\text { heme }\right]^{6+}$. Compared with the solution structure where the heme is indirectly attached to the distal His64 and directly to proximal His93, the gas-phase result suggests that the heme is attached more strongly to a site different from the proximal histidine, presumably to His64. 


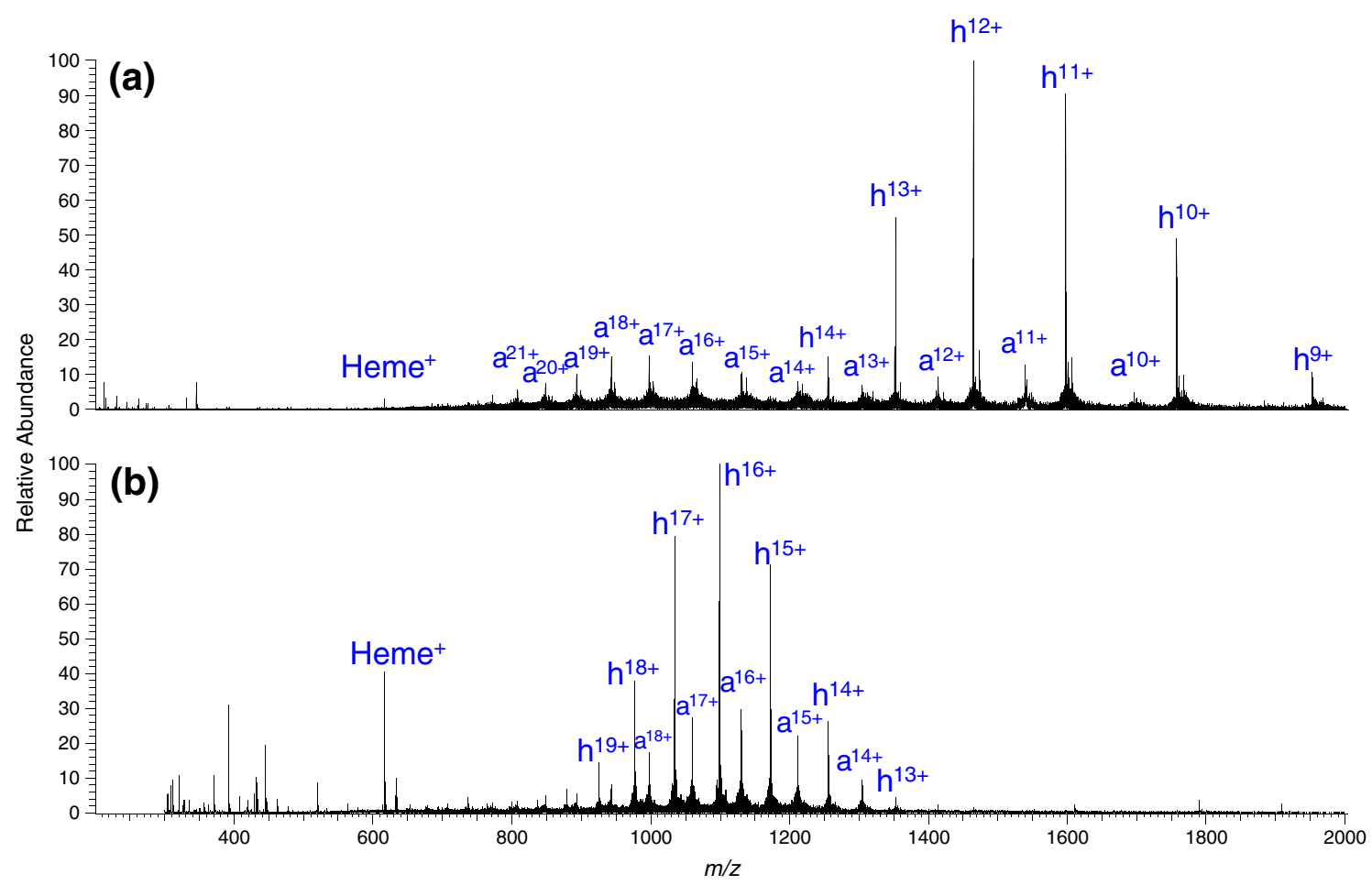

Figure 2. Mass spectra of myoglobin in (a) $3 \%$ methanol/0.5 mM ammonium acetate (b) $5 \mu \mathrm{M}$ myoglobin in $5 \mathrm{mM}$ sulfolane and $30 \%$ acetonitrile. a-apo-myoglobin ions; h-holo-myoglobin ions

In the case of hMb 14+ ions (Figure 4a), the closest to Nterminal heme attachment site is between residues 59 and 97 , as witnessed by the $\left[\mathrm{z}_{94}+\text { heme }\right]^{7+},\left[\mathrm{z}_{94}+\text { heme }\right]^{8+}$, and $\left[\mathrm{c}_{97}+\right.$ heme $^{8+}$ ions (Figure $4 \mathrm{~b}$ ). The fragmentation pattern is consistent with the heme being attached in two positions, i. e., presumably to both His64 and His93.

The ETD spectrum, along with the plot of backbone fragmentation for the $\mathrm{hMb} 16+$, is given in Figure 5. The smallest heme-containing fragments, $\left[\mathrm{z}_{76}+\mathrm{heme}^{6+},\left[\mathrm{c}_{97}+\right.\right.$ heme $]^{9+}$, and $\left[c_{97}+\text { heme }\right]^{10+}$ locate the heme attachment site between residues 77 and 97 . At this charge state the heme seems to be attached in one position, most likely to His93, the proximal group in solution.

\section{Discussion}

The most surprising finding in this work is the fact that in the lowest charge state studied, $12+$, the heme is less strongly attached to the proximal histidine than to another group, presumably the distal histidine. This finding can be accounted for by the following scenario: as discussed in the introduction, in the absence of ligand and distal water, it is likely that the heme becomes six-coordinated, being attached to both proximal and distal histidines. In the absence of interferences from other groups and steric hindrances, the bonding energy to both histidines may be comparable. When ETD occurs with bond fragmentation between these two residues, one of the separating fragments (presumably the one with stronger bonding to heme) will retain the heme group. In multiply protonated $\mathrm{hMb}$, the heme affinity will depend upon the charge state of the respective fragment and the charge location, as the Coulombic repulsion between the heme and the ionizing protons reduces the heme affinity. As the charge state increases, the histidine side chain will eventually become protonated, which is likely to preclude the heme attachment to that histidine.

The information in ETD MS/MS spectra in 12+ ions is not sufficient to draw conclusions on the positions of the ionized protons [21], as many fragments appear in more than one charge state. It is possible, however, that the protonation pattern around the distal histidine and the presence of several basic groups (Figure 1b) that can either be protonated or participate in solvation of the proton localized on a distant residue, will make the heme binding to the proximal His93 less attractive than to the distal His64. Upon further charge increase, the ionizing protons may rearrange and the charge solvation pattern may alter, which could make the proximal His93 relatively more attractive for heme retention than His64. The question that remains unanswered within the current study is whether further reduction in the precursor charge state will change the heme bonding preferentially to the proximal His 93 .

\section{Conclusions}

ETD of intact holo-myoglobin molecular ions provided the heme attachment sites in the gas-phase. These sites were found to be charge-state specific, but broadly consistent with 
(a)

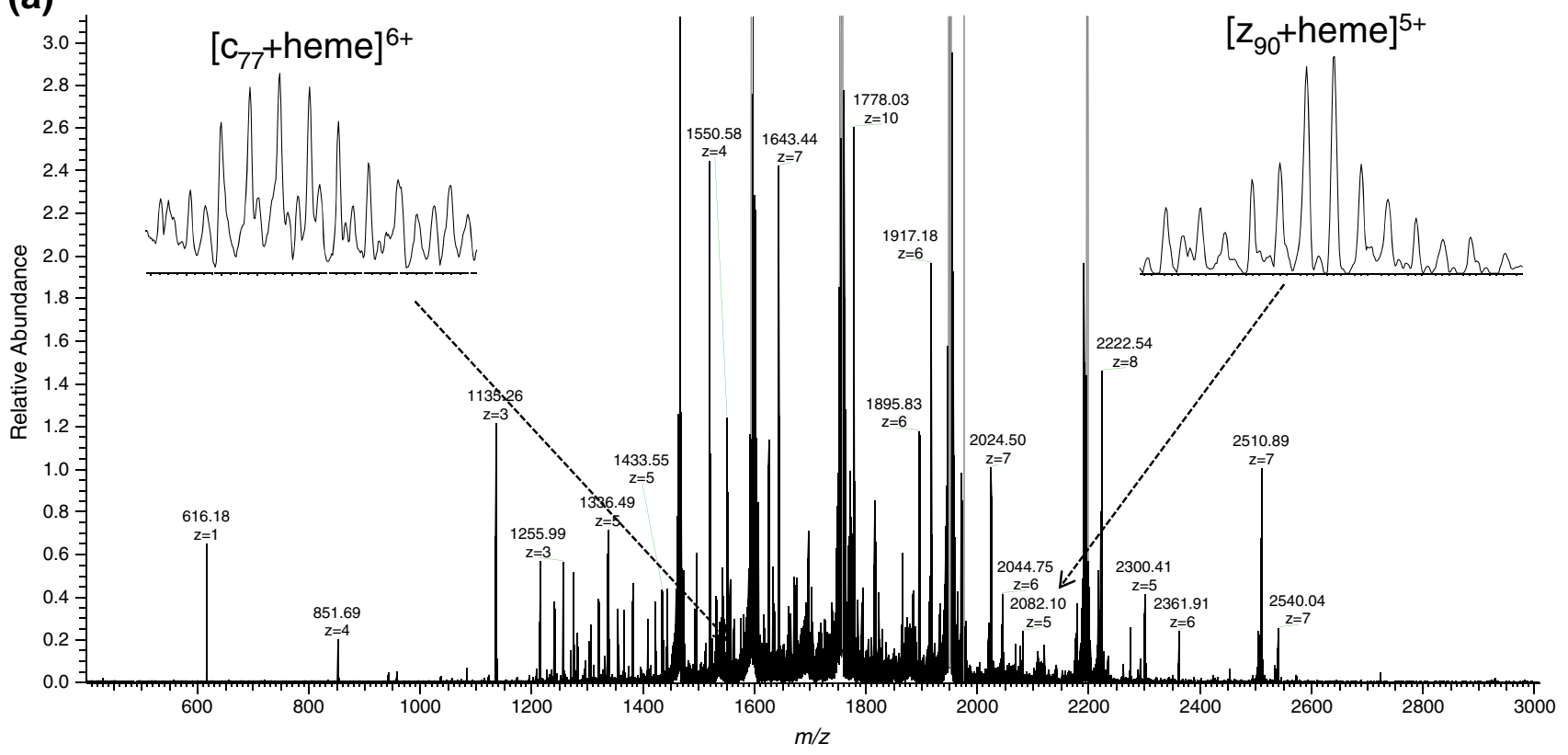

(b)

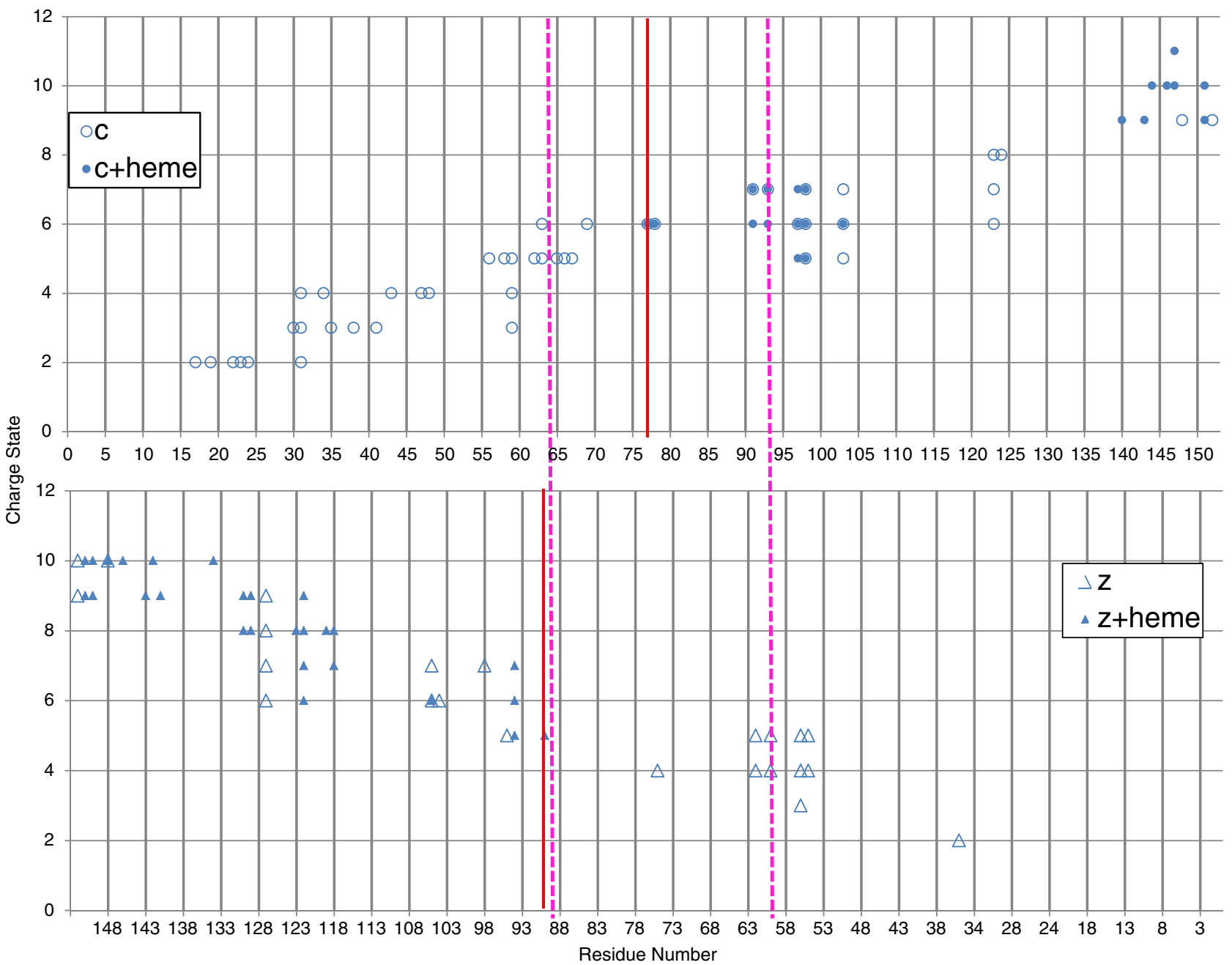

Figure 3. (a) Electron transfer dissociation tandem mass spectrum of 12+ ions of holo-myoglobin (b) Plot of backbone fragmentation against product ion charge state. Thin red line indicates the solution phase heme location and thick purple line refers to the experimentally observed heme location 


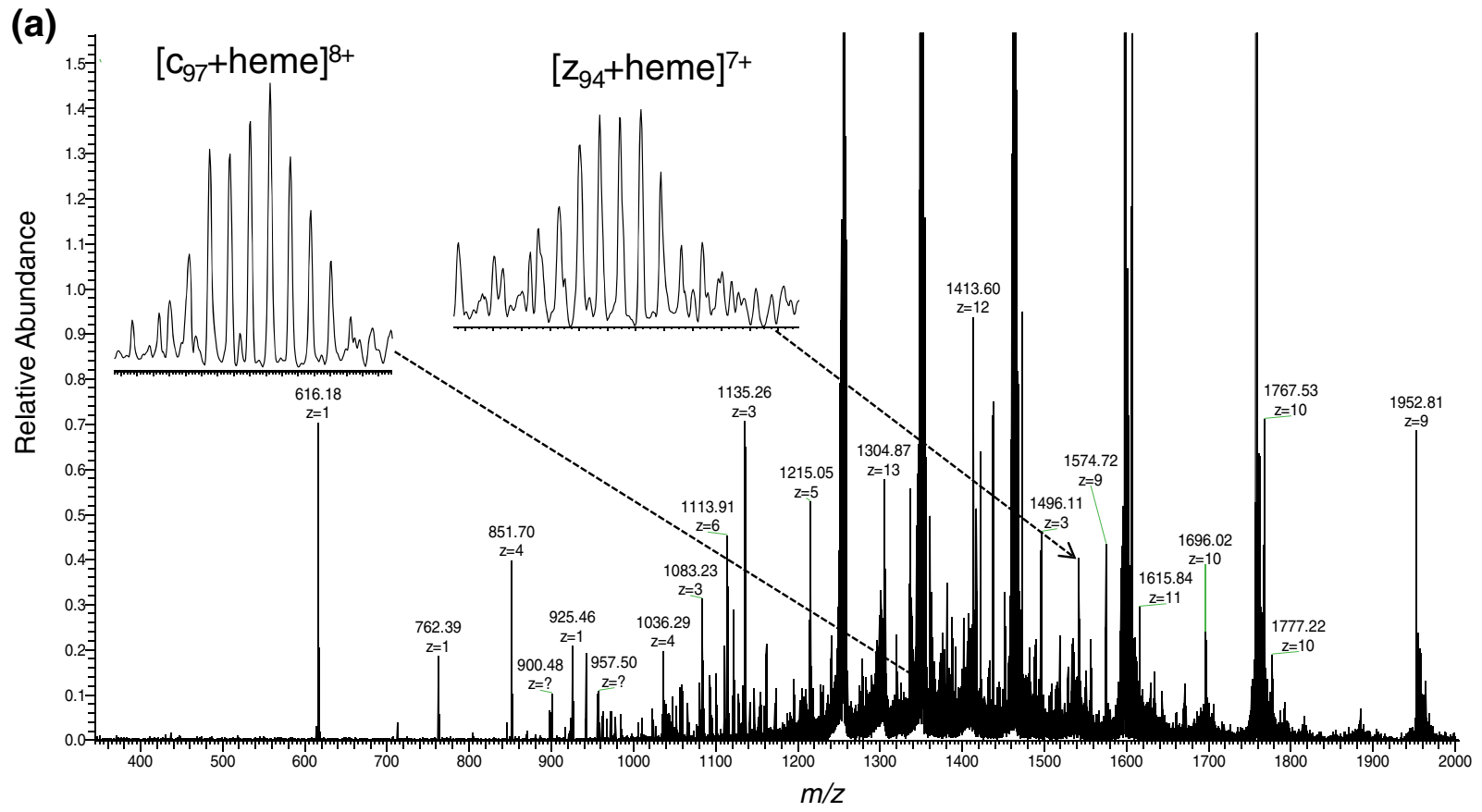

(b)

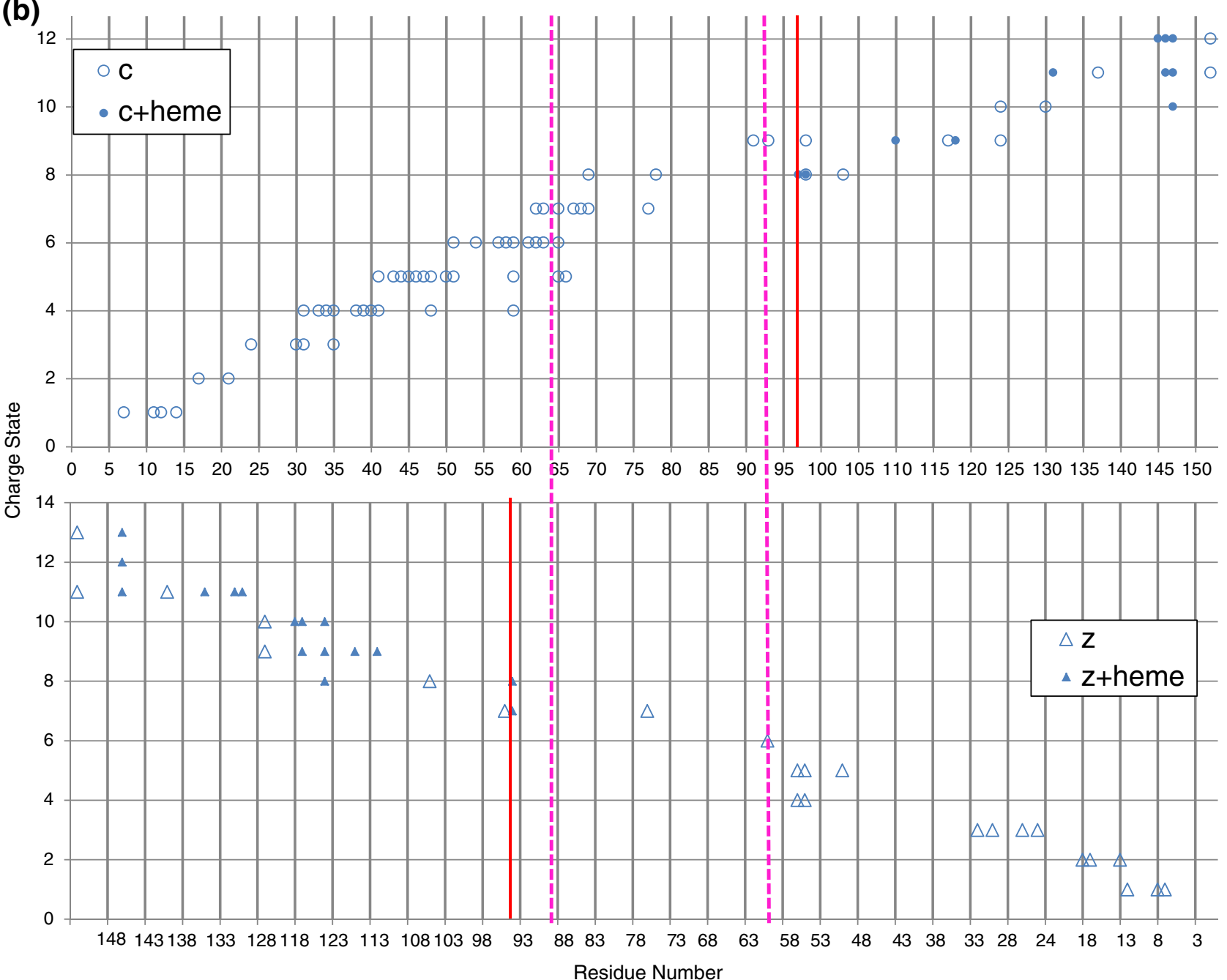

Figure 4. (a) Electron transfer dissociation tandem mass spectrum of 14+ ions of holo-myoglobin. (b) Plot of backbone fragmentation against product ion charge state. Thin red line indicates the solution phase heme location and thick purple line refers to the experimentally observed heme location 
(a)
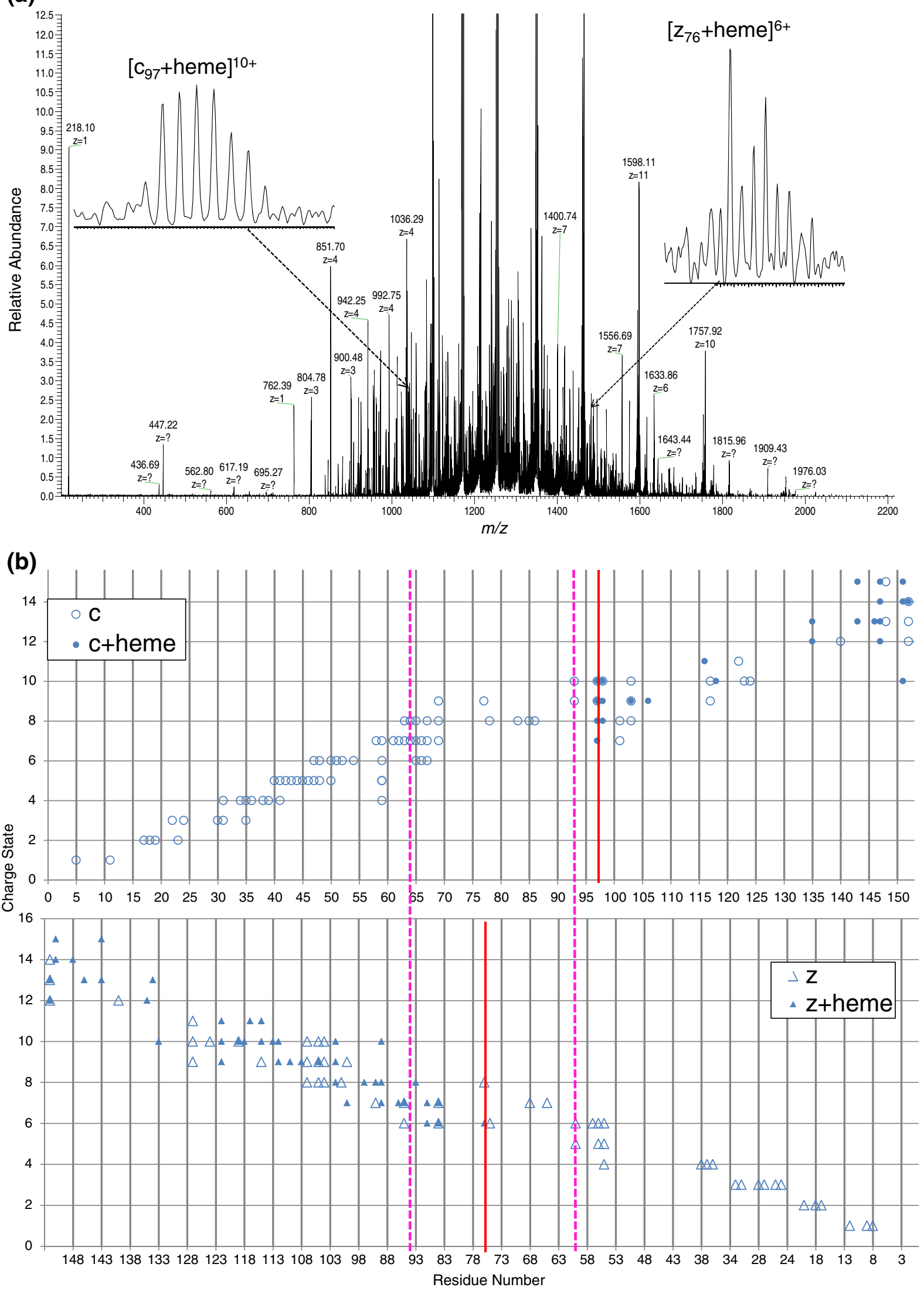

Figure 5. (a) Electron transfer dissociation tandem mass spectrum of 16+ ions of holo-myoglobin. (b) Plot of backbone fragmentation against product ion charge state. Thin red line indicates the solution phase heme location and thick purple line refers to the experimentally observed heme location 
the heme location in solution. However, the preferential heme binding site is charge-state dependent and does not necessarily correspond to that in solution. In particular, in the lowest studied charge state, $12+$, the heme is apparently linked to the distal histidine His64 instead of the proximal one His93, while the situation is reversed in $16+$.

In line with other recent studies (e.g.; [22]), our results further highlight the potential of the ETD/ECD approach in top-down analysis of intact protein-molecule complexes held together by hydrogen and ionic bonds. The results also confirm that the tertiary structure of $\mathrm{hMb}$ can remain relevant to the solution phase upon transition to the gas phase. However, the gas-phase structure is affected by the absence of the distal water molecule and the presence of multiple protonation. Therefore, drawing a link between the gas-phase and solution-phase structures should be done upon careful consideration of these effects.

\section{Acknowledgments}

The authors acknowledge support for this work through grants from the Knut and Alice Wallenberg Foundation and Swedish Research Council (grant 2007-4410 to R.A.Z.). A. A.E. is grateful to Amgen Corporation for support. David Good is acknowledged for careful reading of the manuscript and valuable suggestions.

\section{References}

1. Kendrew, J.C., Bodo, G., Dintzis, H.M., Parrish, R.G., Wyckoff, H., Phillips, D.C.: 3-dimensional model of the myoglobin molecule obtained by X-ray analysis. Nature 181, 662-666 (1958)

2. Harami, T., Kitao, S., Kobayashi, Y., Mitsui, T.: Mossbauer spectroscopic evidence on the heme binding to the proximal histidine in unfolded carbonmonoxy myoglobin by guanidine hydrochloride. Hyperfine Interac. 181, 179-187 (2008)

3. Quillin, M.L., Arduini, R.M., Olson, J.S., Phillips, G.N.: Highresolution crystal-structures of distal histidine mutants of sperm whale myoglobin. J. Mol. Biol. 234, 140-155 (1993)

4. Rifkind, J.M., Abugo, O., Levy, A.: Detection, formation, and relevance of hemichromes and hemochromes. Hemoglobins B 31(Heim, J), 449480 (1994)

5. Riccio, A., Vitagliano, L., di Prisco, G., Zagari, A., Mazzarella, L.: The crystal structure of a tetrameric hemoglobin in a partial hemichrome state. Proc. Natl. Acad. Sci. U.S.A 99, 9801-9806 (2002)

6. Perriman, A.W., Brogan, A.P.S., Colfen, H., Tsoureas, N., Owen, G.R., Mann, S.: Reversible dioxygen binding in solvent-free liquid myoglobin. Nat. Chem. 2, 622-626 (2010)
7. Covey, T.R., Bonner, R.F., Shushan, B.L., Henion, J., Boyd, R.K.: The determination of protein, oligonucleotide and peptide molecular weights by ion-spray mass spectrometry. Rapid Commun. Mass Spectrom. 2, 249-256 (1998)

8. Katta, V., Chait, B.T.: Observation of the heme globin complex in native myoglobin by electrospray-ionization mass-spectrometry. $J$. Am. Chem. Soc. 113, 8534-8535 (1991)

9. Hunter, C.L., Mauk, A.G., Douglas, D.J.: Dissociation of heme from myoglobin and cytochrome b5: comparison of behavior in solution and the gas phase. Biochemistry 36 1018-1025 (1997)

10. Chen, Y.L., Campbell, J.M., Collings, B.A., Konermann, L., Douglas, D.J.: Stability of a highly charged noncovalent complex in the gas phase: Holomyoglobin. Rapid Commun. Mass Spectrom. 12, 10031010 (1998)

11. Schmidt, A., Karas, M.: The influence of electrostatic interactions on the detection of heme-globin complexes in ESI-MS. J. Am. Soc. Mass Spectrom. 12, 1092-1098 (2002)

12. Mark, K.J., Douglas, D.J.: Coulomb effects in binding of heme in gasphase ions of myoglobin. Rapid Commun. Mass Spectrom. 20, 111-117 (2006)

13. Lomeli, S.H., Yin, S., Loo, R.R.O., Loo, J.A.: Increasing charge while preserving noncovalent protein complexes for ESI-MS. J. Am. Soc. Mass Spectrom. 20, 593-596 (2009)

14. Lomeli, S.H., Peng, I.X., Yin, S., Loo, R.R.O., Loo, J.A.: New reagents for increasing ESI multiple charging of proteins and protein complexes. J. Am. Soc. Mass Spectrom. 21, 127-131 (2010)

15. Sterling, H.J., Daly, M.P., Feld, G.K., Thoren, K.L., Kintzer, A.F., Krantz, B.A., Williams, E.R.: Effects of supercharging reagents on noncovalent complex structure in electrospray ionization from aqueous solutions. J. Am. Soc. Mass Spectrom. 21, 1762-1774 (2010)

16. Zubarev, R.A., Kelleher, N.L., McLafferty, F.W.: Electron capture dissociation of multiply charged protein cations. A nonergodic process. J. Am. Chem. Soc. 120, 3265-3266 (1998)

17. Syka, J.E.P., Coon, J.J., Schroeder, M.J., Shabanowitz, J., Hunt, D.F.: Peptide and protein sequence analysis by electron transfer dissociation mass spectrometry. Proc. Natl. Acad. Sci. U.S.A. 101, 9528-9533 (2004)

18. Haselmann, K.F., Budnik, B.A., Olsen, J.V., Nielsen, M.L., Reis, C.A., Clausen, H., Johnsen, A.H., Zubarev, R.A.: Advantages of external accumulation for electron capture dissociation in Fourier transform mass spectrometry. Anal. Chem. 73, 2998-3005 (2001)

19. Xie, Y.M., Zhang, J., Yin, S., Loo, J.A.: Top-down ESI-ECD-FT-ICR mass spectrometry localizes noncovalent protein-ligand binding sites. $J$. Am. Chem Soc. 128, 14432-14433 (2006)

20. Zubarev, R.A., Yang, H.Q.: Multiple soft ionization of gasphase proteins and swift backbone dissociation in collisions with $\leq 99 \mathrm{eV}$ electrons. Angew. Chem.-Int. Ed. 49, 1439-1441 (2010)

21. Kjeldsen, F., Savitski, M.M., Adams, C.M., Zubarev, R.A.: Determination of the location of positive charges in gas-phase polypeptide polycations by tandem mass spectrometry. Int. J. Mass Spectrom. 252, 204-212 (2006)

22. Yin, S., Loo, J.A.: Top-down mass spectrometry of supercharged native protein-ligand complexes. Int. J. Mass Spectrom. 300, 118$122(2011)$ 